\title{
A 4-m evolvable space telescope configured for NASA's HabEx Mission: the initial stage of LUVOIR
}

Charles F. Lillie, Howard A. MacEwen, Ronald S. Polidan, James B. Breckinridge

Charles F. Lillie, Howard A. MacEwen, Ronald S. Polidan, James B.

Breckinridge, "A 4-m evolvable space telescope configured for NASA's HabEx Mission: the initial stage of LUVOIR," Proc. SPIE 10398, UV/Optical/IR Space Telescopes and Instruments: Innovative Technologies and Concepts VIII, 103980R (5 September 2017); doi: 10.1117/12.2274623

Event: SPIE Optical Engineering + Applications, 2017, San Diego, California, United States 


\title{
A 4-m Evolvable Space Telescope Configured for NASA's HabEx Mission: The Initial Stage of LUVOIR
}

\author{
Charles F. Lillie*a ${ }^{\text {a }}$ Howard A. MacEwen ${ }^{\mathrm{b}}$, Ronald S. Polidan ${ }^{\mathrm{c}}$, James B. Breckinridge ${ }^{\mathrm{d}}$
}

${ }^{a}$ Lillie Consulting, LLC, 6202 Vista del Mar, Playa del Rey, CA, USA 90293, ${ }^{b}$ Reviresco LLC, 4901

Loosestrife Court, Annandale, VA, USA 22003, 'Polidan Science Systems \& Technology LLC, 888

SW Evergreen Ave., Redmond, OR, USA, 977564, ${ }^{\mathrm{d} B r e c k i n r i d g e ~ A s s o c i a t e s, ~ L L C, ~}$

985 East California Blvd., Pasadena, CA USA 91106

\begin{abstract}
Previous papers have described our concept for a large telescope that would be assembled in space in several stages (in different configurations) over a period of fifteen to 20 years. Spreading the telescope development, launch and operations cost over 20 years would minimize the impact on NASA's annual budget and drastically shorten the time between program start and "first light" for this space observatory. The first Stage of this Evolvable Space Telescope (EST) would consist of an instrument module located at the prime focus of three 4-meter hexagonal mirrors arranged in a semi-circle to form one-half of a $12-\mathrm{m}$ segmented mirror. After several years three additional 4-m mirrors would be added to create a $12-\mathrm{m}$ filled aperture. Later, twelve more 4-m mirrors will be added to this Stage 2 telescope to create a 20-m filled aperture space telescope. At each stage the telescope would have an unparalleled capability for UVOIR observations, and the results of these observations will guide the evolution of the telescope and its instruments.

In this paper we describe our design concept for an initial configuration of our Evolvable Space Telescope that can meet the requirements of the 4-m version of the HabEx spacecraft currently under consideration by NASA's Habitable Exoplanet Science and Technology Definition Team. This "Stage Zero" configuration will have only one 4-m mirror segment with the same $30-\mathrm{m}$ focal length and a prime focus coronagraph with normal incidence optics to minimize polarization effects. After assembly and checkout in cis-lunar space, the telescope would transfer to a Sun-Earth L2 halo orbit and obtain high sensitivity, high resolution, high contrast UVOIR observations that address the scientific objectives of the Habitable-Exoplanet Imaging Missions.
\end{abstract}

Keywords: Space telescope, coronagraph, exoplanets, on-orbit assembly, servicing, infrastructure, robotics

\section{INTRODUCTION}

Many of the key science questions in astrophysics (e.g. how did the Universe begin and evolve to current state, how do planets form and evolve, and are we alone in the Universe) require very large aperture telescopes. For ground-based observatories there has been a rapid expansion of aperture from the 5 meter Palomar telescope (1948), to the W. M. Keck 10-meter telescopes (1993), to the Thirty Meter Telescope (TMT) and the 39-meter European Extremely Large Telescope (EELT) that are both in development with first light planned for the early/mid-2020s. These giant telescopes have revolutionized and will continue to revolutionize astrophysics.

For space telescopes the evolution of aperture has been much slower than that of ground-based telescopes. From $\sim 1$ meter apertures for the Orbiting Astronomical Observatories (OAO) of the 1960s/1970s, to 2.4 meters for the Hubble Space Telescope (HST) in 1990, to 6.4 meters for James Webb Space Telescope (planned launch in 2018). The primary cause of this slow growth is the fact that these telescopes must be launched into space using a very constraining launch vehicle; current launch vehicle fairings and lift capacity restrict the size of space telescopes to $\sim 9$ meters. Should the Space Launch System (SLS) launch vehicle that is currently being developed by NASA for exploration of the solar system be utilized as a telescope launch vehicle, it would expand the allowable maximum aperture to $\sim 14$ meters, or about $40 \%$ of the aperture of the largest currently planned ground based telescopes.

*charles.lillie@clillie.com; $\quad$ phone 1310 686-8358; $\quad$ fax 1310 827-8194; 
This space telescope aperture constraint can be removed if one abandons the requirement that the telescope be launched fully built in a single large launch vehicle and instead adopt an architecture of assembly of the telescope in space. This approach also improves a wide range other big telescope issues including budget and material issues and provides opportunities for more innovative solutions for future observatories.

This assembly of large telescopes in space architecture has recently been addressed by us (Polidan $e t a l^{1}$ ) and by Lee $e t$ $a l^{2}$ in the Journal of Astronomical Telescopes, Instruments, and Systems (JATIS) 2016 special issue on future large aperture UV/Opt/IR telescopes and applied more generally and with more detail by Boyd et al $2017^{3}$.

\section{WHY ASSEMBLE TELESCOPES IN SPACE?}

The principle reason to assemble astronomical telescopes in space is that astrophysics needs aperture: science will need telescopes much larger than the James Webb Space Telescope (JWST) to obtain measurements with sufficient resolution and signal-to-noise to identify and characterize habitable exoplanets, see planets forming; observe details of galaxy structure, formation, and evolution in the Virgo cluster and beyond; and to look, in detail, at the formation of the first stars and galaxies. The most recent and most complete discussion of the science needs for such large telescopes can be found in the Association of Universities for Research in Astronomy (AURA) "From Cosmic Birth to Living Earths" study ${ }^{4}$, which presents a concept for a 12-meter High Definition Space Telescope (HDST). It is easy to extrapolate from the science goals presented in this study to telescopes much larger than the 12-meter HDST.

To enable space telescopes larger than $\sim 12$ meters we must look to alternative approaches to how space telescopes have been built and launched since the 1960s. Launch vehicles have a size limit, with the Saturn V and the current Space Launch System (SLS) representing the largest reasonable launchers; similarly, fairings cannot be infinitely large. These lift and volume capacity constraints force us to adopt an in-space assembly architecture if we are to have 16-meter (and larger) space telescopes.

Attempting to achieve the science goals of finding examples of life outside the solar system and understanding the details of the formation of planets, stars, and galaxies will be very difficult and very expensive if we restrict ourselves to "small" ( $\sim 40 \%$ the size of their ground-based counterparts) space telescopes. We must advance a new and innovative architecture i.e., an Evolvable Space Telescope (EST). EST would be constructed in space from subcomponents that would be launched in smaller launch vehicles, assembled in space, and evolve over time from a smaller initial stage to a 20-meter or larger aperture. It would be regularly serviced and upgraded as was done with the Hubble Space Telescope (HST), performing science measurements continuously, as with HST, except for servicing/upgrading intervals.

Fortunately, the science need for bigger telescopes is being enabled through a large number of technology and other developments in launch vehicles, robotics, and general space infrastructure ${ }^{5}$. These include the development of much lower cost medium-lift launch vehicles that could move us to a very different system-level cost curve, continued advancement of robotic/telerobotic capabilities, and the deployment of a concept and program for a cis-lunar, longduration human-occupied "deep space gateway" by NASA.

In addition, proceeding down the assembly in space path brings in a large number of additional benefits ${ }^{3}$. The pronounced budget peaks that, in the current flat-budget era, often delay or stop other science initiatives can be modulated to flatten the peaks and accommodate budgetary ups and downs for maximum efficiency. As demonstrated with HST science instrument replacement, in-space upgrades greatly expand the science return; and coupling the instrument upgrades with aperture upgrades will expand the science return of the observatory even more. New technologies can easily be planned, developed, and incorporated into the observatory, and the costs (manufacture and testing) of using robust materials and designs to survive launch loads can be greatly reduced. The system level science and cost benefits of an Evolvable Space Telescope will enable an affordable path to 20+ meter telescopes in space that is not achievable utilizing traditional approaches of launching a fully assembled telescope on a single launch vehicle.

\section{EVOLVABLE SPACE TELESCOPE CONCEPT DEVELOPMENT}

The Evolvable Space Telescope (EST) concept development was in initiated at Northrop Grumman Aerospace Systems in 2014 (see Reference 1 and the citations within for a full history) to enable a path to large space telescopes. 
Three characteristics particularly distinguish the EST mission concept from other approaches: Evolvability, adaptability, and serviceability.

- Evolvability. This, of course, is the core of the EST concept, and directly implies that there will be several configurations of EST as it evolves in several "stages" over the observatory's lifetime. These stages will be separated from each other by several years (nominally five) and will provide substantial performance advances based upon the evolution of the science drivers and/or available technologies. The Stage 1 EST will be launched as a fully functional observatory designed to produce compelling science. Subsequent stages increase the telescope collecting area, add new instruments, and/or upgrade telescope and spacecraft subsystems. Stage 1 will be designed to provide a significant capability to address the science drivers currently being used to define HDST, and it will form the core of future even larger telescopes, beginning with the collecting area of an equivalent $\sim 7-\mathrm{m}$ telescope and evolving to a 16- to $20-\mathrm{m}$ aperture telescope at Stage 3.

- Adaptability. The EST concept can respond to changing conditions outside control of the program itself. Many external effects are quite obvious; for example: budgetary changes (either negative or positive), science priority changes that occur during telescope and instrument development, and technical advances in areas such as detectors/instruments, optical systems and coatings, structural materials, disturbance control, robotic servicing, and other important areas.

- Serviceability. This capability is essential to each of the preceding two, since it directly addresses the program's ability to maintain performance against failures or wear and tear, major or minor, and the ability to enhance or upgrade systems, again including minor enhancements or stage changes. It is also directly related to the continued operation of the EST over many decades, lasting perhaps as long as 50 or more years with periodic robotic and/or crewed servicing.

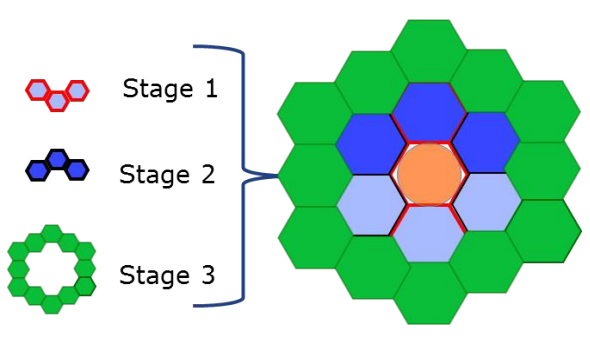

Figure 1. The evolution of EST's Primary Mirror Assembly from the initial three 4-m (flat-to-flat) hexagonal segments of the offaxis Stage 1 EST to the eighteen mirror segments of the $20-\mathrm{m}$, on-axis, f/1.5 filled aperture Stage 2 EST.

This nominal evolution of the EST primary mirror assembly (PMA) is described below and shown pictorially in Fig. 1. Illustrations of the full observatory in each of its three stages are presented in Fig. 2.

- EST Stage 1 is indicated in Fig. 1 by the three gray hexagons and the central circle. The hexagons form the initial Primary Mirror Assembly (PMA), and will typically be formed to the master prescription. The central circle represents the prime focus instrument module (or secondary mirror), and will be designed to serve the same role throughout Stages 1, 2, and 3. These elements will all be orbited and assembled using the first EST launch, providing an off-axis aperture on the order of $12 \mathrm{~m} \times 4.5 \mathrm{~m}$ with a primefocus instrument suite. In this concept, Stage 1 is designed to be an exceptional UV astrophysics observatory with its large $\left(40-\mathrm{m}^{2}\right)$ collecting area, low scattered light, off-axis configuration, and high transmittance to the prime-focus instruments. It could also accommodate a Vis-IR coronagraph and wide-field camera.

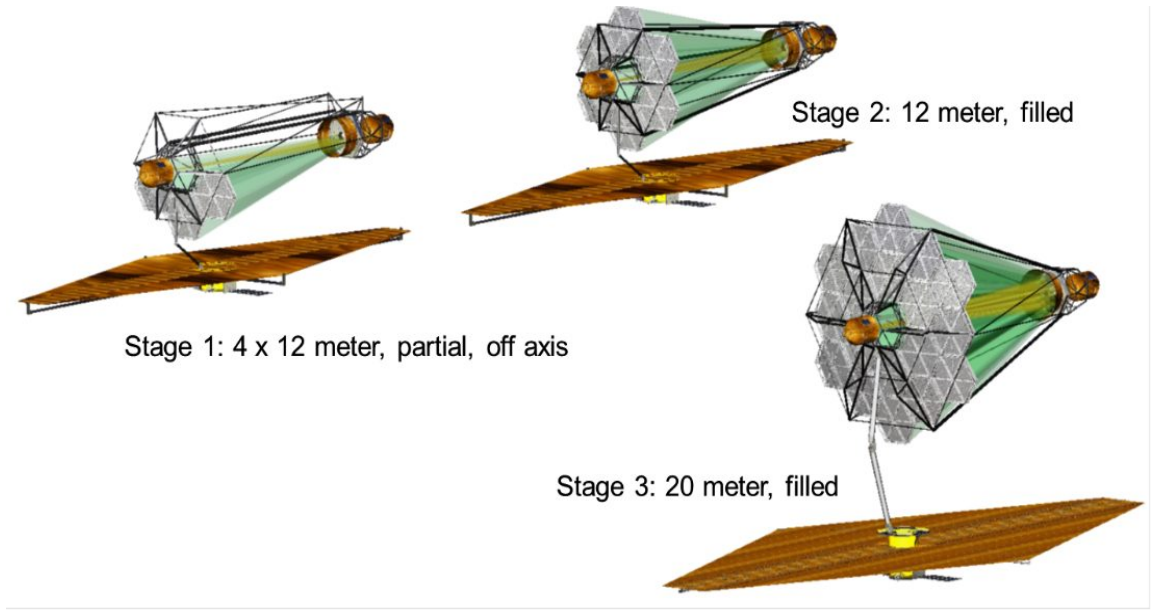

Figure 2. The full EST Observatory in each of its three stages as it evolves from a $4 \mathrm{x}$ 12 meter off-axis telescope with a $40-\mathrm{m}^{2}$ collecting area to a 12-meter on-axis telescope with an $80-\mathrm{m}^{2}$ collecting area, and then to a 20 -meter on-axis telescope with a $240-\mathrm{m}^{2}$ collecting area. 
- EST Stage 2. At the chosen time (about half a decade following Stage 1 deployment), three additional segments (shown in dark blue) will be launched, along with other components (e.g., new instruments and additional tensegrity truss structural elements) to augment the Stage 1 observatory and form the Stage 2 EST: a 12-m filled aperture space telescope with a prime-focus and/or a Cassegrain or three mirror anastigmatic (TMA) configuration.

- EST Stage 3. Again, approximately a half-decade after the launch of the Stage 2 components, the next stage of telescope evolution will occur, in this case, adding 12 mirror segments (in green) to form a $20-\mathrm{m}$ aperture for the telescope in a Cassegrain configuration. In this case, since the size of the PMA will be significantly increased, the upgrade will include modification or replacement of the sunshield, and structural modifications and servicing as needed.

- EST Stage 4+. Over its 40 to 50 year lifetime the EST can continue to grow beyond the 20 -meter aperture and/or evolve in other ways, utilizing the latest technology developments to address new science requirements.

\section{A PRECURSOR TO EST FOR THE HABEX MISSION}

In this section we describe a precursor mission to that of the Evolvable Space Telescope described above. This Stage-0 EST would be utilize many components of the Stage-1 EST, but the observatory configuration would optimized to achieve the scientific objectives of the HabEx mission; and its primary scientific instrument would be a high-throughput coronagraph for exoplanet imaging and spectroscopy.

This Stage-0 EST would have only one of the three 4-m hexagonal segments of the Stage-1 EST primary mirror, and the metering structure between the primary and the Prime Focus Instrument Module (PFIM) would be a tensegrity truss similar to the optical bench for the initial EST design (Polidan, et al, 2014) ${ }^{6}$. The spacecraft bus and the positioning boom that connects the bus with the telescope would be the same as those for the Stage-1 EST, but a conformable "sugar-scoop" sunshield would be used instead of the planar sunshield of the later stages of EST. The Stage-0 EST would, however, be designed for augmentation at a later date with additional mirror segments and instruments and the planar sunshield of EST Stages 1,2 and 3.

The optical design for the Stage-0 EST is shown in Figure 3. It features a 4-m, f/7.5 off-axis telescope with a Lyot Coronagraph located at its prime focus. The primary mirror of the telescope a hexagonal mirror that will, in the future, be one of the six segments of the 12-m on-axis telescope of the Stage-2 EST (and the inner ring of the 20-m, 18 segment Stage-3 EST).

The coronagraph is located in an enclosure in one of the four quadrants of a $3.8-\mathrm{m}$ diameter, 5-m long PFIM. The PFIM is centered on the optical axis of the (future) Stage-2 EST $\sim 30$-m from the primary mirror, $\sim 5-\mathrm{m}$ beyond the place where a Cassegrain secondary mirror might be located in the future as EST evolves. The coronagraph utilizes the entire volume of the instrument enclosure to maximize the separation between optical elements in order to minimize the curvature of the optical services and polarization effects. The coronagraph design is described in detail in Section 5.

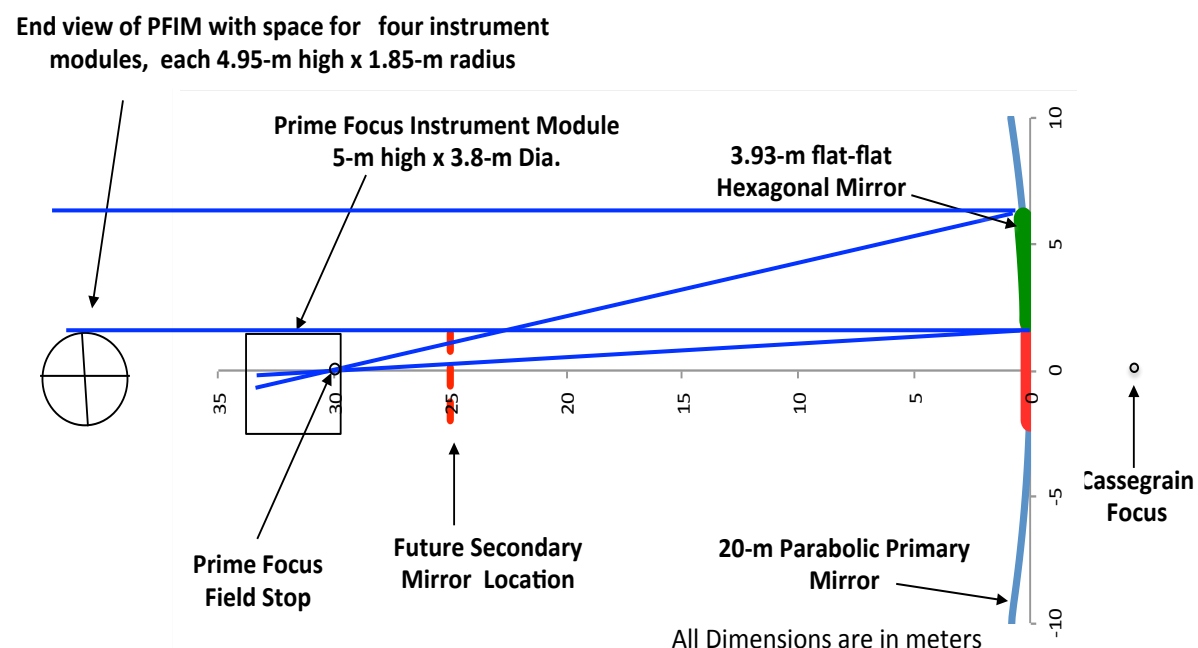

Figure 3. The EST Stage-0 optical design showing one 4-m, f/7.5 hexagonal segment of the 20-m EST Stage- 3 primary mirror located $30-\mathrm{m}$ from the Prime Focus Instrument Module that houses a Lyot Coronagraph. Also shown is the place where a Cassegrain secondary mirror could be located in the future, as well as the location of the Cassegrain focus. 
The instrument enclosure that houses the coronagraph is shown in Figure 4. The enclosure is designed for on-orbit removal and replacement, with guide rails for alignment, blind mating electrical connectors kinematic latches. The EST instrument enclosure have design heritage from the instrument enclosures developed for the Advanced X-ray Astrophysics observatory (AXAF) ${ }^{\mathrm{i}}$ which is now the Chandra X-ray Observatory). AXAF was designed for on-orbit servicing in low earth orbit with the space shuttle. Removal and replacement of these enclosures was demonstrated in the Water Emersions Test Facility (WETF) at Marshall Space Flight Center. ${ }^{7}$
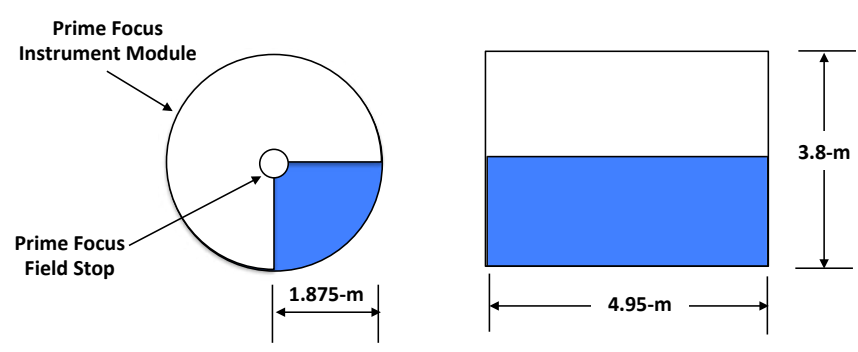

Figure 4. The instrument enclosure that houses the Stage-0 EST coronagraph is shown in blue. The PFIM has accommodations four four instruments that can be removed and replaces on-orbit.

\section{Our EST Stage-0 Configuration}

for the HabEx mission is shown in Figure 5. This conceptual design has a high degree of heritage from previous studies for future space observatories, technology developments efforts with internal funds, and exiting flight hardware. For example the spacecraft bus design is derived from the JWST spacecraft (TRL 7); the PFIM and the instrument module were developed for AXAF (TRL 5); the tensegrity truss was developed for the international X-ray Observatory

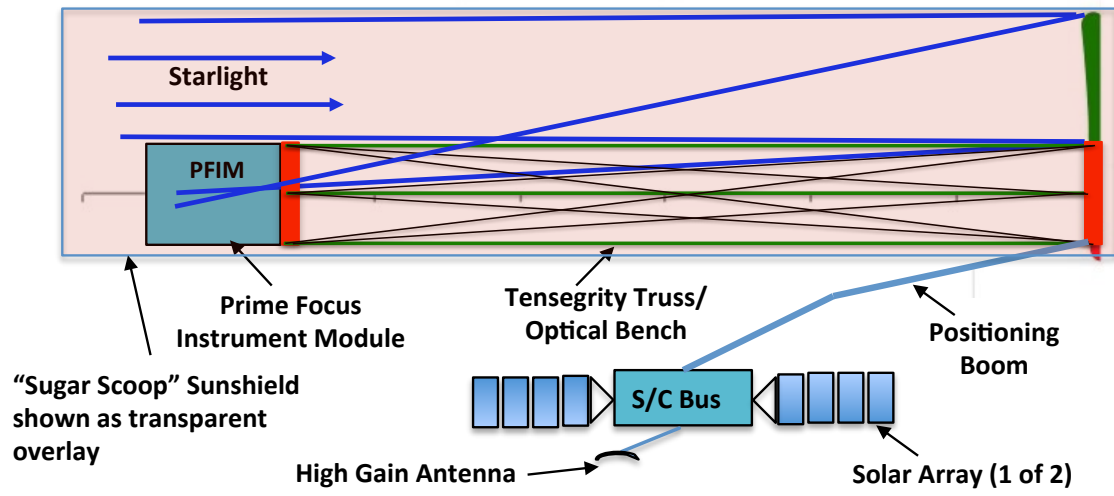

Figure 5. The EST Stage-0 configuration optimized the for HabEx mission. (IXO) mission (TRL 3); the sugar-scoop sun-shield (Figure 6) was designed for the Terrestrial Planet Finder Coronagraph (TPF-C) mission and developed for other missions (TRL 4); and the positioning boom (which provides a 2pi field of regard for EST) was designed (and patented) for SAFIR (the Single Aperture Far Infrared) telescope and other future space observatories (TRL 2). Segmented primary mirrors for large telescopes, and the hardware and software to successfully align and phase the segments has been demonstrated both on the ground (Keck, SALT, LAMP) and for space (JWST) (TRL 8); and efforts are on-going to develop the large (4-m), lightweight mirrors and the high reflectivity mirror coatings required for EST (TRL 3-4).

Stage-0 Mission Concept. We envision the Stage-0 EST as a precursor mission designed to operate in an SEL2 halo orbit for approximately five years using its UV/VIS/NIR coronagraph to detect and characterize nearby exoplanets. Space is available in the PFIM for three other instruments, such as a UV imaging spectrometer and a wide field VIS/NIR imaging spectrometer for general astrophysics observations if sufficient funding were available.

The Stage-0 EST would be launched into cis-

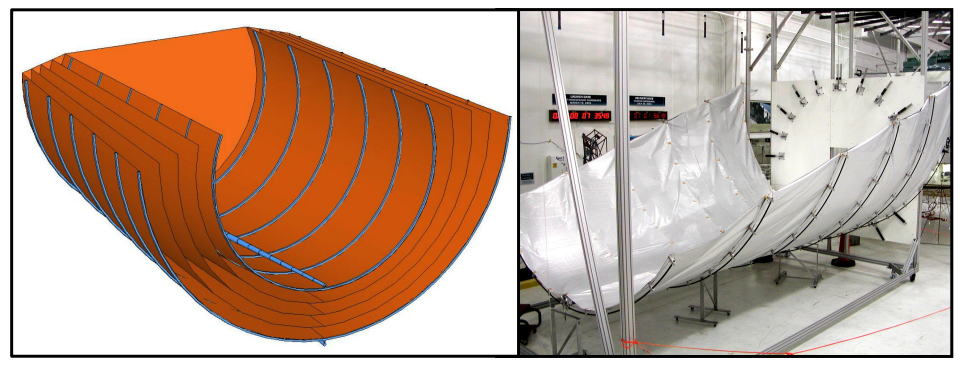

Figure 6. The Multi-layer Sugar Scoop sunshield designed to provide microKelvin thermal stability for the TPF-C telescope (on the left) and the Prototype sunshield developed by Northrop Grumman (on the right).

\footnotetext{
${ }^{\mathrm{i}}$ In order to reduce cost AXAF was redesigned in 1992. In the process, its orbit was changed from a Low Earth Orbit similar to that of HST to a highly elliptical 16,000 x 133,000 Kilometer orbit, with no servicing options. For additional detail see https://en.wikipedia.org/wiki/Chandra X-ray Observatory
} 
lunar space with a large launch vehicle such as the Falcon 9 Heavy, Vulcan or New Glenn and rendezvous with a Deep Space Gateway (DSG) in a halo orbit (ideally) around the Earth-Moon L1 or L2 point. ${ }^{\text {ii }}$ At the DSG the Stage-0 EST would be deployed and/or assembled robotically (or telerobotically. All components of the EST would be designed for assembly and servicing with dexterous manipulators mounted on robotic arms. Astronauts aboard the DSG would most likely control these robots, and Extra-Vehicular Activities (EVA) by astronauts would be reserved for unforeseen contingencies and emergencies.

After being assembled and checked out at the DSG, the Stage-0 EST would use its internal propulsions system to insert itself into a transfer orbit toward SEL2. Instrument commissioning would occur during cruise, so that mission operations could commence shortly after insertion into its SEL2 halo orbit.

Figure 7 shows a tentative schedule for the EST program. It assumes studies of the EST mission concept will be conducted in the mid-2020s with a new start in 2028, and a 60-month development schedule for the EST Stage-0 HabEx mission, with launch and first light in 2033 or 2034. Every five years thereafter the components for the next Stage of EST would be launched into cislunar space (or to SEL2, depending on the infrastructure available) where they would be added to the existing EST observatory to enhance its capabilities. Thus over a period of $\sim 15$ years EST would evolve from a 4-m f/7.5 off-axis telescope for HabEx in 2033 to a 4 x 12-meter LUVOIR telescope in 2038; a 12-m f/2.5 onaxis filled aperture telescope in 2043; and a 20-m, f/1.5 filled aperture, on-axis LUVOIR telescope in 2048 or 2049.

Alternatively, a $12-\mathrm{m}$ (or 15-m) HDST/LUVOIR telescope could be developed in the traditional way: design, built, integrate, test, launch (with SLS), and deploy (autonomously) on-orbit during transit to an SEL2 halo orbit. As with HST, Chandra, an most recently JWST, ambitious initial designs and cost estimates will lead to budget growth, schedule slip, and a descope resulting in reduced aperture and other capabilities. With NASA's consistently flat budget, the optimum 7 or 8-year development schedule for the next Great Telescope will undoubtedly be stretched to $\sim 15$ (or 20) years with a "standing army" of workers for twice as long, for about twice the "shouldcost" in Real-Year Dollars (RY\$) of an optimum development schedule. Thus with a new start in 2028, a 12-m HDST aka LUVOIR) is unlikely to be launched before 2043, with first light in 2044.

As shown by Figure 7, assembly (and servicing) the next large space telescope in space and evolving it in several stages over time to increase its capabilities has several distinct advantages when compared to the traditional approach for space telescope development:

\section{Space Telescope Size vs. Time}

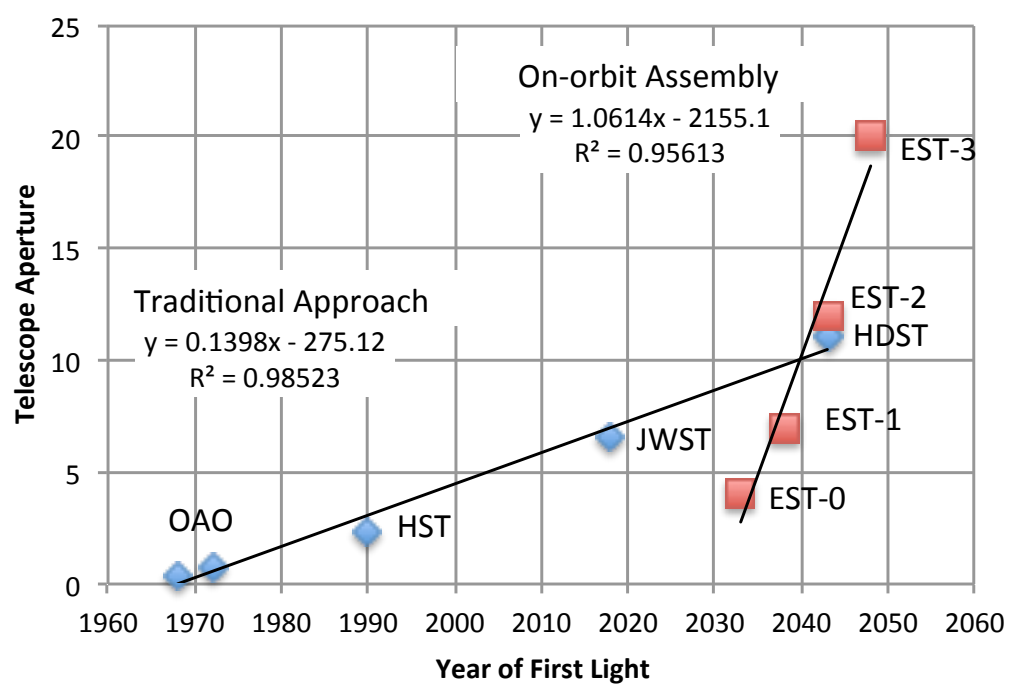

Figure 7. This graph shows the year in in which NASA's previous UVOIR space telescopes reached orbit versus their effective aperture along with the projected launch date of a 12-m HDST, assuming it is developed with the traditional approach with a new start in 2028. The expected launch date for four EST Stages is also shown, assuming the same new start date with assembly in cis-lunar space.

\footnotetext{
${ }^{i i}$ NASA is currently considering several DSG orbits. In order by their accessibility for assembly and servicing of a large space telescope, they are the L2 Halo, Distant Retrograde, Near-Rectilinear Halo, Elliptical Lunar and Low Lunar orbits. The DSG would have the ability to change its orbit for different mission activities.
} 
- A 4-m EST-0 (HabEx) observatory could return cutting-edge observations of ExoPlanets 10 years before an HDST could reach orbit;

- A 4 x 12-m EST-1 (LUVOIR) observatory would have first light 5 years before HDST;

- A 12-m EST-2 1 (LUVOIR) observatory would be in its SEL2 at the same time as HDST (with newer technology, 10-years of operational experience and science discoveries, and a very experienced workforce);

- A 20-m EST-2 1 (LUVOIR) observatory would be operational at SEL2 by 2049 ( 62 years before a 20-m telescope could be developed using the traditional approach, if the current trend continues).

In addition, the adaptable design of EST make it possible to modify the various EST configurations to meet new requirements that result from budget changes, new technologies, new in-space infrastructure, and new science objectives; the technology used for HDST would be 15 to 20 years old when it was launched, compared with the five to 8 -year old technology for each EST stage; and, even neglecting the impact of inflation on RY\$, the current $>\$ 1 \mathrm{~B}$ cost of a SLS would easily cover the cost of four large launch vehicles for an EST.

Finally, we note that the aperture of EST is not limited to 20-meters. A third ring of 4-m mirror segments could be added to EST-3 to create a $28-\mathrm{m}$ f/1.07 on-axis, filled aperture telescope for an EST Stage-4 LUVOIR Great Observatory.

\section{INSTRUMENTS FOR AN EVOLVABLE SPACE TELESCOPE}

The evolvable space telescope architecture offers astronomers a revolutionary platform for scientific discoveries in the mid-twenty first century. Unlike JWST, the EST architecture includes an innovative capability for in-space replacement of instruments in response to changing scientific priorities and new technology. The EST provides astronomers a very large facility not unlike the CERN Hadron particle accelerator where different scientific groups gather instruments to make fundamental physics discoveries. In the case of EST, there is a very large re-usable space aperture-facility for all qualified scientists to bring their instruments to make astrophysical discoveries.

In this Section, we examine a possible suite of instruments that may satisfy the mid-century (2050) astronomical science measurement objectives. These are: a high performance Lyot coronagraph; a high Étendu integrated optics spectrometer; and an ultraviolet spectrometer.

The prime focus instrument spacecraft package is shown in Figure 4 as a cylinder of dimensions 3.8 meters in diameter and 5 meters in length. Here we divide the cylinder into four sectors for field-sharing the instruments in the manner used by the Hubble Space Telescope ${ }^{\mathrm{i}}$ (HST).

\subsection{Lyot Coronagraph}

Figure 8 shows a cartoon of the classic Lyot coronagraph. The telescope mirrors are shown as lenses (optical power) for ease of understanding. This system was first shown to be useful for exoplanet characterization in a 1982 paper by Breckinridge, Kuiper and Shack. ${ }^{8,9}$

In Figure 8, light enters from the left to reflect from the large primary mirror (represented here as a lens). The EST primary is an example. Because the field of view is so narrow there is no need (at the EST prime focus F\#) for either secondary or tertiary optics to deliver a diffractionlimited image at the image plane stop. The complex occulting mask is located at the image plane stop. Following the light path further we see that a collimator is positioned so its front focus is super-posed on the occulting mask to create an image of the telescope exit pupil superposed onto a complex amplitude \& phase Lyot stop, whose function is to control the diffracted light that surrounds the exit pupil. Further to the right in the system, another mirror (lens) is placed to relay the electric field at the complex image plane stop as modified by the complex occulting mask onto a detector. The modulus squared of the complex vector field is recorded at the detector. Missing from this

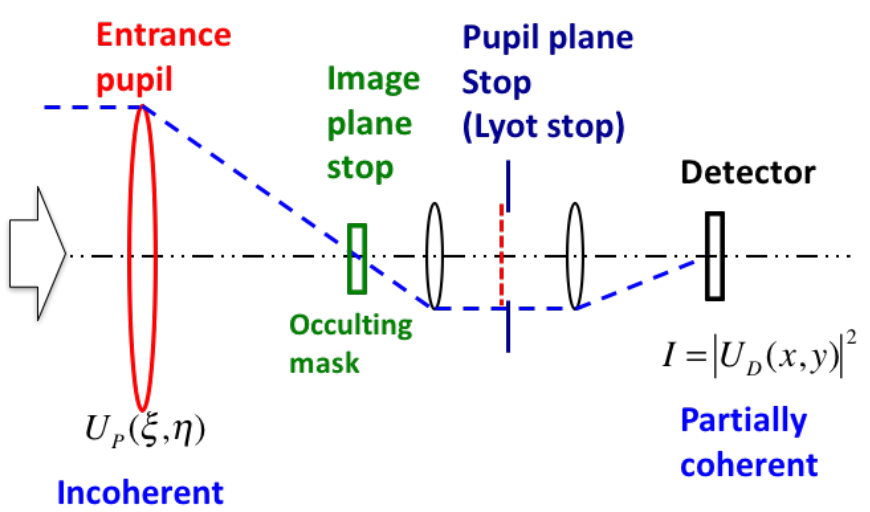

Figure 8. A cartoon showing a classical Lyot coronagraph with lenses substituted for mirrors for ease of understanding. 
cartoon are the mirrors necessary to create the Talbot ${ }^{10,11}$ fringes, and thus provide another level of control to the complex vector wavefront. We discuss those two mirrors in the next section.

The Lyot coronagraph for EST-0 utilizes the single 4-meter f/7.5 primary mirror segment for the entrance aperture. The Lyot coronagraph is one of several high contrast starlight suppression systems that are candidates for direct imaging and spectroscopy of exoplanets in the optical and near infrared. Other candidates are the shaped pupil ${ }^{12}$, vector vortex ${ }^{13}$, nulling ${ }^{14}$, apodized aperture ${ }^{15}$, and phase induced amplitude apodization ${ }^{16}$ coronagraphs, along with many others. Much research continues today to identify the optimum coronagraph opto-mechanical and physical optics design that maximizes the productivity of space-based telescope/coronagraph systems. One of the many challenges has been to match the vector electromagnetic white light (partially coherent) electric field to a high-dynamic range complex occulting mask (that can be fabricated) for maximum extinction of unwanted starlight and minimum extinction of the nearby very faint exoplanets which are separated by as little as $2 \lambda / \mathrm{D}$ radians from the centroid of the $\operatorname{star}^{17,18}$. Spacecraft structures and dynamics and current launch vehicle fairings constrain space-telescope apertures larger than 4-meters to be segmented as we show in Figure 5 for EST. Diffraction from the edges of these segments provides a particular challenge to the design of coronagraphs ${ }^{19}$. In this paper, we present a first-order geometrical optics design for a classic Lyot coronagraph as updated by new technology ${ }^{20,21}$ to correct the quasi-static wavefront errors we expect from the evolvable space telescope (EST).

Internal polarization effects and light scattered from mirror surfaces create unwanted noise on the exoplanet signal. To minimize these effects, we minimize the mirror count and design a 5-mirror-surface coronagraph. If we include the primary mirror the total number of reflections is six.

Figure 9 presents the first-order optomechanical layout for a low $\mathrm{F} / \#$ coronagraph with no unnecessary fold mirrors and therefore a minimum number of reflecting surfaces. Both of these aspects, combined with properly designed mirror coatings will minimize polarization aberrations for the end-toend system. This prime-focus instrument system collects radiation directly from the 4-meter primary (M1). Radiation strikes M2 where the image plane stop is collimated. It then passes on to reflect

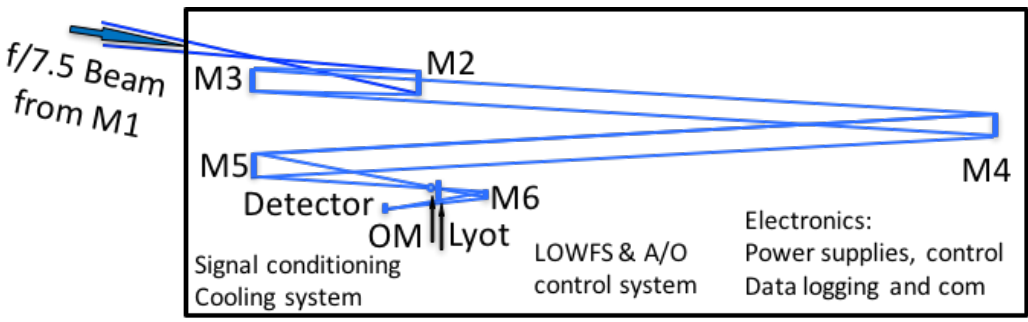

Figure 9. First-order opto-mechanical layout for the EST six-mirror coronagraph for high contrast imaging of exoplanets The detector and other electronics are also shown in their relative locations, where they can be accessed during instrument integration and testing, and servicing on-orbit. from two deformable mirrors (M3 and M4).

These two mirrors form the "Talbot" fringe pair. The light is then reflected to an off-axis parabola (M5) where a relayed image of the image plane stop falls on the occulting mask (OM). Progressing further along the optical path takes us to an image of the 4-meter entrance aperture where the Lyot filter is placed to block the unwanted radiation that diffracts around the entrance pupil (the 4-meter primary mirror). The off-axis parabola on mirror M6 is paired with M5 to form an image of the exoplanet on the Detector for Nyquist sampling.

The entire instrument, including electronics for signal conditioning and processing, the A/O sensing and control system, including the Low Order Wave Front Sensor (LOWFS), cryogenics and power supplies all fit within a 90-degree sector of the 3.8-meter diameter x 5-meter long instrument cylinder. This enables three other instruments in field sharing mode to fit within the instrument module.

Table 1 lists the first-order optical prescription for the opto-mechanical layout shown in Figure 9 above. Column 1 identifies the optical element, column 2 gives the design of the mirror (OAP for offaxis paraboloid and DM for deformable mirror), column 3 shows the diameter in meters, column 4 presents the focal length in meters, column 5 gives the $\mathrm{F} / \#$ for the
Table 1. First order optical prescription for the EST coronagraph with a 16 arc-second field of view (FOV) for general astrophysics and a 6 arcsecond FOV for ExoPlanet observations

\begin{tabular}{|c|c|c|c|c|c|}
\hline Mirror & Type & Size $(\mathbf{m})$ & $\begin{array}{c}\text { Focal } \\
\text { Length }(\mathbf{m})\end{array}$ & f/no. & $\begin{array}{c}\text { Separation }(\mathbf{m}) \\
\mathbf{M n} \text { to } \mathbf{M n + 1}\end{array}$ \\
\hline M1 & OAP & 4.000 & 30.000 & 7.500 & 31.012 \\
M2 & OAP & 0.1307 & 0.980 & 7.500 & 1.012 \\
M3 & DM & 0.1280 & Flat & & 4.500 \\
M4 & DM & 0.1280 & Flat & & 4.501 \\
M5 & OAP & 0.1307 & 0.980 & 7.500 & 1.403 \\
M6 & OAP & 0.0436 & 0.208 & 4.771 & \\
\hline
\end{tabular}


mirror, and column 6 shows the separation between optical surface vertices.

Several important aspects of this design are listed below:

- Six mirror system provides $>60 \%$ transmittance;

- M3 and M4 are 128 x 128 actuator Xinetics DMs;

- 512 x 512 pixel EMCCD photon counting detector with 16-micron pixels;

- Inner Working angle of 83 milli-arcseconds (mas) and Outer Working Angle of 1320 mas at $400 \mathrm{~nm}$;

- Enhanced Silver or $\mathrm{AlF}_{3}$ mirror coatings for 400 to 950 or 90 to $950 \mathrm{~nm}$ bandpass;

- 6 arc second Field of View for Exoplanets, 16 arc second FOV for general Astrophysics; and

- Focal Ratios $\geq 4.7$ minimize polarization effects to maximize image quality for $10^{-10}$ contrast

This design is very flexible and only minor changes in the focal length of M6 are needed to either decrease or increase the science field of view (FOV). The scale of the image plane at the occulter can also easily be increased or decreased by increasing or decreasing the focal length of M5.

The optical layout for the coronagraph is optimized by having no obstructions or shadows on the pupil, which reduces the diffraction noise to only that from the light diffracting around the edges of the 4-meter aperture. The number of reflections is minimized and the curvature on all powered elements is a minimum, which reduces Fresnel polarization.

\subsection{Integrated Optics Spectrometer}

As astronomical telescopes grow in size, general-purpose classical optical/IR imaging spectrometers and integral field spectrometers grow in size to match the optical Étendu required to avoid vignetting and the consequent loss of power across the focal plane. We see this in ground-based observatories, where the volume and mass of the instruments for the next generation of large aperture telescopes keeps increasing. Yesterday's telescopes are the mass and volume of today's instruments. Clearly, we need to break this growth curve and use innovative modern photonics to create smaller cost-effective instruments with the same science gathering capability of larger, more massive and expensive instruments. One of the technologies that will create this needed breakthrough in volume, mass and cost is the integrated optics spectrometer, or "spectrometer on a chip". ${ }^{22,23}$

The spectrometer function takes place within a sheet of solid-state integrated optics located close to or in contact with a focal plane. Each pixel has its own moderate to high-resolution spectrometer. The challenges of this architecture are radiometric calibration and transmittance. The power that falls on each pixel will pass into its own integrated optics miniature spectrum analyzer. The absorption needs to be minimal. If not the power collection advantage of the large aperture is lost. But the factor of over $10^{3}$ gain in less mass and volume may overwhelm the power-loss factor to create a productive cost effective imaging spectrometer (integral field spectrometer). This technology has been demonstrated on brass-boards ${ }^{22,23,24}$ and these Astro-photonics instruments will be available in the 2025 time frame for the EST mission.

\subsection{Ultraviolet Spectrometer}

The ultra-violet region of the spectrum between 90 and $200 \mathrm{~nm}$ region of the spectrum contains lines from the groundstate transitions for most of the elements in the Universe. Access to this region of the spectrum is important to study elemental abundances in the Universe as well as astrophysical processes important to evolutionary tracks of stars, exoplanets and galaxies.

The Far Ultraviolet (FUV, $120-200 \mathrm{~nm}$ ) and Lyman Ultraviolet (LUV, $91.2-121 \mathrm{~nm}$ ) are unique bands. Solid-state physical processes, in particular the interaction of photons at energy levels around $100 \mathrm{~nm}$, cause significant absorption and loss of signal. This is shown in Figure 10, which is taken from the SPIE 9122 paper by Moore, Hennessey, Jewell, Nikzad and France. ${ }^{25}$

The title of their figure reads "Theoretical calculations predicting the LUV reflectance of Al capped with 2 nm ALD $\mathrm{AlF}_{3}$ (solid line), $\mathrm{MgF}_{2}$ (dashed line) and $\mathrm{LiF}$ (dash-dotted line) with no interfacial oxide layer. All three outperform bare $\mathrm{Al}$ that has been exposed to air and developed a 2-nm native oxide layer of $\mathrm{Al}_{2} \mathrm{O}_{3}$ (dotted line), but the $\mathrm{ALD} \mathrm{AlF}_{3}$ film 


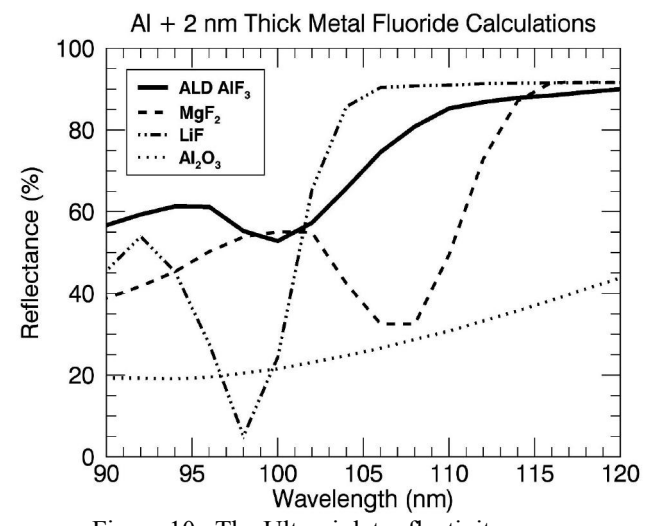

Figure 10.. The Ultraviolet reflectivity versus wavelength of four Far- UV mirror coatings: $\mathrm{ALD} \mathrm{AlF}_{3}, \mathrm{AlMgF}_{2}, \mathrm{LiF}$, and $\mathrm{Al}_{2} \mathrm{O}_{3}$.

has the highest average reflectance in the LUV. This plot exemplifies the potential of ALD $\mathrm{ALF}_{3}$ as a future LUV optimized coating and has proven to maintain broadband optical performance out to $800 \mathrm{~nm}$."

Figure 9 shows that the coating reflectivity is about $58 \%$ from $\lambda=90$ to $102 \mathrm{~nm}$, after which it increases to $85 \%$ at $110 \mathrm{~nm}$ and reaches $90 \%$ at $120 \mathrm{~nm}$. The telescope cost algorithm given in Equation TBD below is approximate. Stahl ${ }^{26}$ provides an in-depth analysis to show that the exponent can vary between approximately 2.0 and 2.5 depending on technology maturity and other factors. For our discussion here, we select an exponent of 2.2 and describe the cost as a function of aperture in Eq. 1.

$$
C \propto A_{T}^{2.2}
$$

Where $C$ is cost and $A_{T}$ is the aperture of the telescope. Breckinridge and Lillie $^{27}$ used this relationship to calculate the increase in telescope cost as a function of mirror count and mirror coating reflectivity which reduce the effective area and aperture/diameter of the telescope. These costs are shown in table 2 below.

Table 2. Growth of the telescope diameter and cost required to maintain constant photon flux as a function of reflectivity and the number of mirrors in a telescope/instrument system for $\mathrm{R}=0.58,0.85,0.90,0.98$ and the No./Mirrors $=1$ to 6

\begin{tabular}{|c|cc|cc|cc|ccc|cc|c|}
\hline No.of Mirrors & \multicolumn{2}{|c|}{1} & \multicolumn{2}{|c|}{2} & \multicolumn{2}{|c|}{3} & \multicolumn{3}{|c|}{5} & \multicolumn{3}{c|}{6} \\
\hline $\mathrm{R}(\%)$ & Dia. & Cost & Dia. & Cost & Dia. & Cost & Dia. & Cost & Dia. & Cost & Dia. & Cost \\
\hline 0.58 & 1.00 & 1.00 & 1.30 & 1.79 & 1.69 & 3.19 & 2.21 & 5.70 & 2.87 & 10.19 & 3.74 & 18.21 \\
0.85 & 1.00 & 1.00 & 1.08 & 1.20 & 1.18 & 1.43 & 1.28 & 1.71 & 1.38 & 2.04 & 1.50 & 2.44 \\
0.90 & 1.00 & 1.00 & 1.05 & 1.12 & 1.11 & 1.26 & 1.17 & 1.42 & 1.23 & 1.59 & 1.30 & 1.79 \\
0.98 & 1.00 & 1.00 & 1.01 & 1.02 & 1.02 & 1.05 & 1.03 & 1.07 & 1.04 & 1.09 & 1.05 & 1.12 \\
\hline
\end{tabular}

For example, if the UV end-to-end optical system design has 3 mirrors, with $58 \%$ reflectivity on each surface, then to maintain the detector plane flux we would have with only one mirror results in a cost increase factor of 3.19. Clearly a prime focus system like EST is optimum for UV astrophysics in the 90 to $102 \mathrm{~nm}$ range.

Based on the analysis given above we seek to find UV imaging and spectrometer designs that have 1 or at most 2 reflections. The UV causes some particular issues with the primary mirror and the level to which it must be kept clean and free of any contamination. The best strategy may be to maintain only one of the 4-meter segments with a far UV mirror coating and mask the exit pupil to see only that particular segment.

The 4-m EST provides an opportunity to design a single reflection UV imaging system for minimal UV absorption or a Rowland Circle spectrometer which has 2 reflections, counting the primary to it's detector.

\section{ON-ORBIT ASSEMBLY AND SERVICING}

For several reasons, not the least of which is the Congressional mandate ${ }^{28}$, all proposed space observatories such as the candidate Habitable Exoplanet Imaging Mission, the HabEx Stage-0 system must be serviceable to sustain long operating lifetimes and replenish consumables. This political mandate is strengthened by the value of the data that could be lost due to a system malfunction and replacement cost.

Moreover, if serviceability is coupled with accessibility, the achievable science can be greatly magnified by system upgrades and replacement of instruments, as done with the Hubble Space Telescope (HST) in five servicing missions. ${ }^{29}$ An example of HST servicing is shown in Figure 11, with an astronaut on a manipulator arm mounted on the Space Shuttle. Of course, the first servicing mission (SM-1) was probably the most important, since it included correction of an optical error in the HST primary mirror that had initially prevented the telescope from working as designed, while later servicing both enabled HST to achieve a lifetime longer than originally expected (still

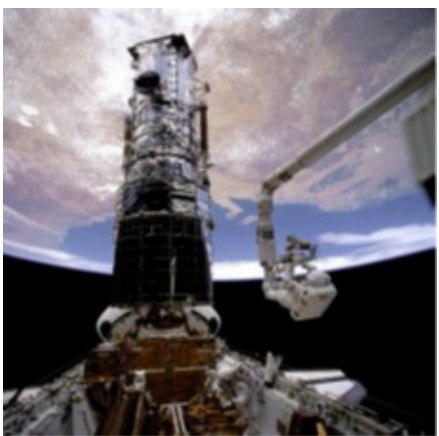

Figure 11. Servicing HST Courtesy: NASA 
not ended!) and dramatically increased the science capability of the observatory. While the HST servicing missions can certainly be regarded as highly successful, they were also assembly missions that included switching out older instruments and inserting ${ }^{\mathrm{iii}}$ newer, powerful instruments. This represented, therefore, continued assembly of the HST system, and highlights the ambiguity and haziness of the division between assembly and servicing missions in general.

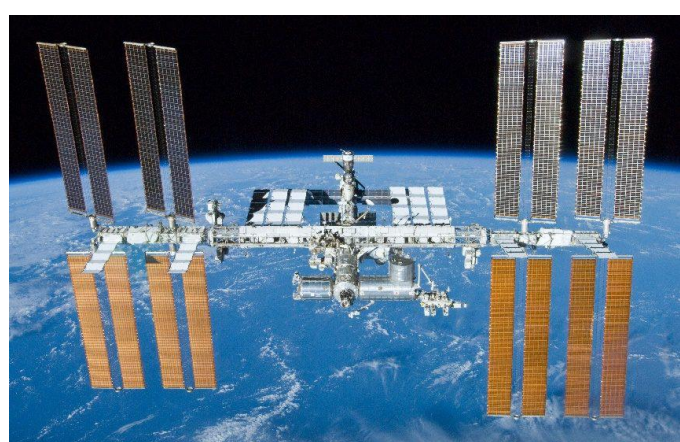

Figure 12. International Space Station (ISS) Courtesy: NASA

As another example, the International Space Station (ISS) was totally dependent upon assembly ${ }^{30}$ from a myriad of separate components and subsystems, many of which then required further servicing (either by crew, robots, or both). The results of this years-long process are illustrated in Figure 12.

HST and the ISS are examples of orbital systems that required both assembly and servicing (including improvisation using available parts and materials) for useful and successful missions, with servicing notably dominant for HST, and assembly dominant of the ISS. They do not in any way exhaust the past examples of in-space assembly and servicing ${ }^{31,}{ }^{32}$, but are sufficient to establish the value of the capabilities involved, and other cases are well documented in the literature.

\subsection{Current Active Development}

Many studies are currently addressing in-space servicing and assembly, under government, commercial, and joint sponsorship, including in-space manufacturing (i.e., fabrication of small parts, not just assembly of manufactured components). Given the wealth of material available ${ }^{32}$, we only mention two initiatives ${ }^{\text {iv }}$ of the US Government: the NASA Restore-L program ${ }^{33}$ for servicing of low altitude satellites (with spinoff to deep space servicing ${ }^{34}$ ) and the DARPA Robotic Servicing of Geosynchronous Satellites (RSGS) ${ }^{35}$. An early artist's concept of Restore-L is shown in Figure 13. Both programs are in active development assisted by industrial partners, and, if funded, both are expected to lead to deployment of commercial servicing systems.

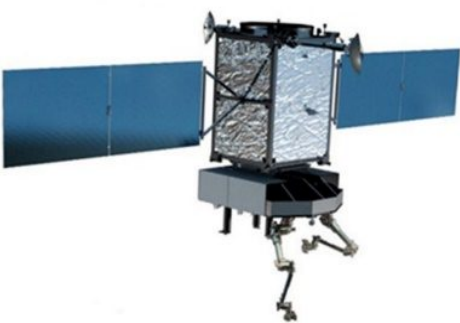

Figure 13. NASA Restore-L Demonstrator Concept Courtesy: NASA

The 4-meter Stage-0 EST designed for the HabEx mission is expected to require only a limited amount of servicing, and assembly assistance limited to observation, with perhaps some capability to ensure precision of deployment of the telescope system ${ }^{\mathrm{v}}$. As telescope systems grow in response to more demanding science problems, assembly will increasingly replace on-ground manufacture and in-space deployment for launch of large telescope systems.

Servicing, as distinct from assembly, is likely to be very limited for systems in for the next few years until the Orion capsule and commercial servicing vehicles are available. Until the mid 2020s it will likely be limited to replenishment of fluids (generally propellants) for station-keeping and lifetime extension and a limited capability to remove and replace a few subsystems (e.g., batteries, attitude controls) or scientific instruments. All of this will require little more than careful design of the original spacecraft in line with interoperability standardization, plus an ability to either transfer the serviced spacecraft or the servicer to a rendezvous point.

\subsection{Developments for the Future}

The coming generation of large space telescopes (following JWST and WFIRST) will almost certainly be the last generation assembled on the ground, tested pretty much as a complete vehicle, and launched and deployed using a single

iv The immediate future of these two programs will not be clear until submittal of this manuscript, when the 2018 Budget is submitted. It is possible, for example, that the two could be combined into a single program.

${ }^{v}$ This may provide a convincing argument for accompaniment of the telescope to its deployment site by a crewed Orion spacecraft equipped with a small complement of tools. 
launch vehicle. ${ }^{\mathrm{vi}}$ For several reasons, most importantly the lack of any launch vehicle that will be able to lift their volume (even folded) and mass, they will have to be manufactured on the ground, launched in component/subsystem form, and assembled in space either in LEO, on the way, or in final orbit. Moreover, recent studies (for example, by the Institute for Defense Analysis [IDA] ${ }^{36}$ and by the Center for Strategic and International Studies $[\mathrm{CSIS}]^{37}$ ) have indicated that this approach will lead to a substantial reduction in system life cycle costs, largely since the unassembled telescope will be packaged in more robust configurations for launch, will not have to withstand massive launch loads in a complex structure, and will not need extensive testing in launch configuration.

\subsection{Launch Vehicles}

The single most important element of the in-space infrastructure is clearly the launch vehicle. This is also the element over which the astrophysics community has essentially no control - we must select from what will be available at launch time. At the time of this writing, there are three basic classes of launch vehicles to consider: Very Large, Large and Medium.

1. Very Large. This class has a single representative, the Space Launch System ${ }^{38}$, or SLS, already in development (although the first flight will not occur until after the SPIE meeting). While there are a number of SLS variations (mostly differentiated by the presence or absence of a crew capability), we will assume that the largest of the class will be used to launch a large space telescope: that is, the SLS Block II Cargo, advertised as having a launch capability to cis-lunar space of 50 metric tons (MT). In the telescope design, its capability will define the largest space telescope that can be launched, fully folded, in a single launch and then deployed in deep space. Larger telescopes that may develop later will require several launches and assembly in space, as noted earlier. The SLS is illustrated in an artist's concept in Figure 14. It is important to note that the production rate for the SLS will be low $(\sim 1$ or 2 per year $)$ and, since there is no other comparable vehicle in the foreseeable future, the loss of a single launch would indefinitely delay a launch campaign for large space telescopes - if not completely terminating it.

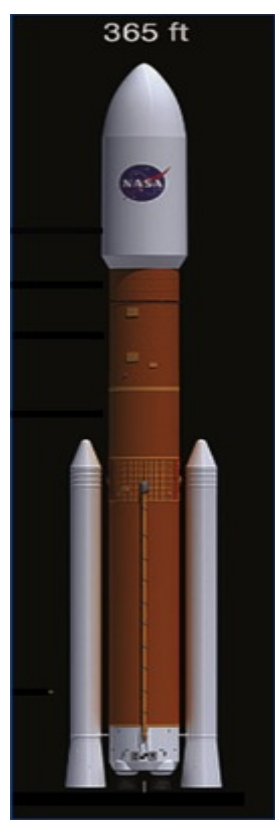

Figure 14. SLS Block 2 Cargo Courtesy: NASA

2. Large. In contrast to the SLS, there will be several vehicles of this class, and some of them are currently operational with excellent reliability records. The known mix includes the Delta IV-Heavy ${ }^{39}$, Ariane $5 \mathrm{ECA}^{40}$, Falcon Heavy ${ }^{41}$, Vulcan, and New Glenn, with others as unknown possibilities: the first three of these are shown in Figure 15, to approximate relative scale (the Delta IV is approximately 269 feet tall). None can lift a 20 -meter aperture telescope to

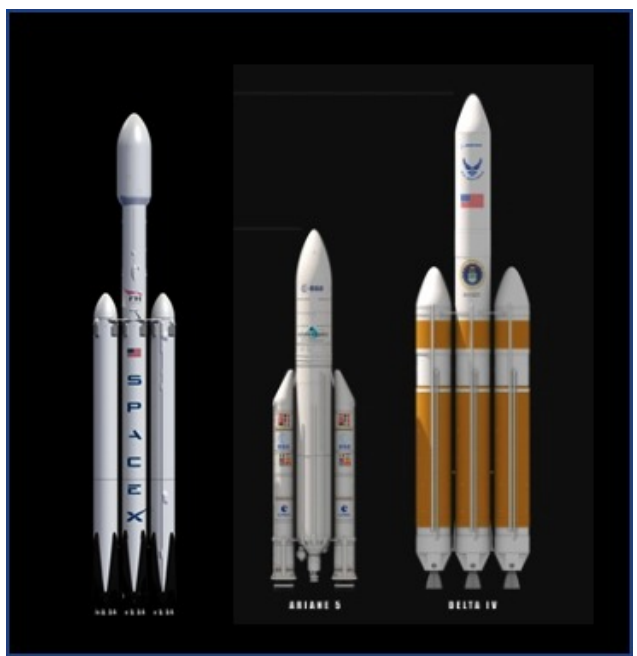

Figure 15. Current Large Launch Vehicles: Falcon Heavy, Ariane 5, \& Delta IV Heavy Courtesy: NASA SEL2 halo orbit in a single flight, but they are all capable of deploying such a vehicle in two or three flights, given on-orbit assembly. While orbital capabilities to cis-lunar space are not advertised, they all can transfer between 15 and 25 Metric Tons (MT) to Geosynchronous Transfer Orbit (GTO). Assuming a 60\% efficiency between GTO and SEL2 halo (the value of the Ariane 5 ECA mission for JWST), this will enable payload deliveries between 10 and 15 MT per launch for assembly into a large space telescope.

It could be highly advantageous to have the extra flexibility in a launch program that multiple launch vehicles and assembly provides, since the loss of a single flight is not necessarily totally catastrophic to the science mission. Care in timing the ground assembly and launch schedules could enable the whole mission to survive a single launch disaster, albeit with a severely altered launch and science collection schedule, which is not a possibility if the entire program is riding on a single launch.

3. Medium. Launch vehicles such as the Falcon 9, Atlas V, Antares and Delta IV Medium could also play an important role in the assemble and servicing of space telescopes in cis-lunar space by delivering up to $\sim 6200$

vi An approach that will remain appropriate for small spacecraft, of course. 
$\mathrm{kg}$ of cargo to a gateway in an Earth-Moon L1 halo orbit in a single flight. These vehicles provide responsive, reliable access to space at costs per kilogram that have significantly decreased with the advent of reusable launch vehicles and the resultant price competition.

\subsection{Space Stations}

Following launch vehicles, the next most important element of the infrastructure is likely to be space stations, of which two classes can be identified: large, permanently crewed stations in Low Earth Orbit (LEO), and one or more smaller stations (perhaps only crewed during periods of actual use) that may be positioned in cislunar space.

1. Large Stations. This class of stations is currently represented by the International Space Station (ISS). The ISS can play an important, but limited, role in the evolution of large space telescopes, not as an assembly and departure point, but as an experimental station to investigate the space environment and its effects on space systems and crews. It may also serve as a holding point, or depot, for spare parts and/or consumables high in the Earth's gravity well

2. Smaller Cislunar Stations. These stations, or "Gateways" ${ }^{42}$, are currently in the early mission and performance study program phase, as mandated by Congress. An early example of a Gateway concept is shown in Figure 16. They would be crewed only as necessary (for example, with crews of 4 to 6 arriving on an Orion or non-US craft for stays of weeks to months or longer), and would rely heavily on the Lagrangian points and halo orbits in the Earth-Moon system (EML1 and EML2, in particular) due to the very low energies needed for transfers among such halo orbits ${ }^{\text {vii }}$. Large space telescopes could additionally take advantage of halo orbits around equivalent Lagrange points in the Sun-Earth system (SEL2) ${ }^{\text {viii }}$, also benefiting from a very low transfer energy requirement.

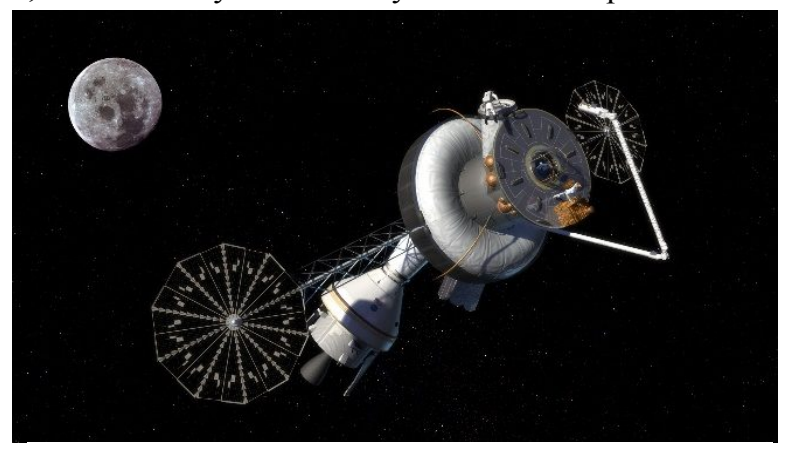

Figure 16. Deep Space Gateway Concept. Courtesy: NASA

These Gateways, far removed from LEO, could provide assembly and servicing for large space telescopes. And, due to the low transfer energy requirements, if servicing an LST stationed at SEL2, they could either transfer themselves to the telescope, or it could be transferred to the $\mathrm{EML}^{\mathrm{ix}}$ halo orbit of the Gateway and then returned once servicing was completed. If assembly or servicing was performed in a crewed configuration at SEL2, the crew would not travel on the Gateway, given the extended flight time and exposure to hazards. Rather, a high-energy transfer stage (e.g., Orion with a chemical boost stage) would be required both for the route out and for the return, adding significantly to the cost.

In addition, it will clearly be necessary for the Gateways to have some means of manipulating the telescopes, their spacecraft, and supporting equipment. As shown in Figure 16, two means of doing this are long manipulator arms (already familiar from use on the Space Shuttle and the ISS) and external, unconnected systems. In Figure 16 the spacesuited person shown working outside the Gateway represents the external system. Given the risk and difficulties of human EVAs, however, alternative systems should be considered.

\subsection{Servicing Vehicles}

While many assembly and servicing missions may be possible using only remote manipulator arms from a Gateway Station, as these missions increase in complexity it will become essential to increase flexibility and responsiveness by employment of separate servicing spacecraft that, while perhaps tethered to the Gateway, will operate as if free-flying in near formation ${ }^{\mathrm{x}}$ with the Gateway and the serviced spacecraft. These servicers will take a variety of forms, ranging

\footnotetext{
vii Several lunar orbits are also being considered, of course, as discussed previously in section 4.

viii Note that Heliophysics also can benefit from an equivalent halo around the inner Lagrange point SEL1.

${ }^{\text {ix }}$ Either EML1 or EML2 is equally useful for the telescope, and can be selected in collaboration with other missions.

$x$ 'Near' being defined based upon each individual scenario.
} 
from large spacecraft ${ }^{\mathrm{xi}}$ (perhaps on a similar scale as the Gateway) to small free-flying robots to crewed single person spacecraft ${ }^{43}$ (SPS - see Figure 17) to individual astronauts whose "spacecraft" would simply be their spacesuit. Vehicles as small as cubesats could be used for (among other applications) close inspections and metrology to ensure high quality of servicing by other spacecraft, and there may even be roles for highly miniaturized 'Chipsats' to repair electronic systems, modify spacecraft surfaces to enhance thermal control and replace failed solar cells, or the like.

\subsection{Observations and Future Path}

We offer three principal observations:

1. For the upcoming generation of large astrophysics space telescopes, assembly and servicing systems and technologies currently available are probably sufficient to support an affirmative development decision in roughly 2025, with deployment occurring in the mid-2030's. However, significant funding for detailed engineering of these capabilities will need to be included in the budget requests. xii

2. For the succeeding generation of large space telescopes that will be driven by still more difficult science issues uncovered in the intervening years and designed for deployment in the period 2045 through 2050, it will not be possible to construct a launch vehicle much larger than the SLS, almost certainly for programmatic reasons and possibly for structural reasons as well. Therefore, multiple launches and in-space assembly of these telescopes will become an

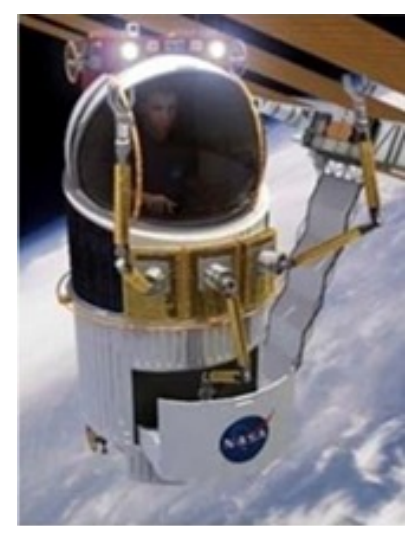

Figure 17: Single Person Spacecraft. Courtesy: NASA unavoidable necessity. To achieve this, development of in-space servicing technologies, systems, and infrastructures needs to begin now.

3. These systems and capabilities will benefit far more space science, development, and exploitation stakeholders than just the astrophysics community. Indeed, work is already proceeding along principal development lines, but it is essential to ensure that the needs of astrophysics are met. Therefore, development must be sponsored, funded, and conducted by a consortium of all identifiable stakeholders involved in space science, exploration, development, and exploitation.

\section{DISCUSSION}

Astronomers always need more collecting area and higher spatial and spectral resolution to detect and characterize the faint, distant stars, nebulae, galaxies, exoplanets and other celestial bodies in order to advance our understanding of our universe. Since Galileo first observed the heavens with a 26-mm telescope in 1609 ground-based telescopes have grown to the 10-meter Keck telescopes of the 1990s, with the 24.5-m GMT ${ }^{\text {xiii }}, 30-\mathrm{m}$ TMT and 39-m EELT scheduled for first light in the 2020s. Space Telescopes have followed a similar progression, growing in size from the 20 -cm photometers of OAO in 1964 to the 2.4-m HST and the 6.5-m JWST, with on-going studies for 16-m (and larger) space telescopes.

The aperture of space telescopes developed in the traditional manner is constrained by the size of current launch vehicle 5 -m payload fairings to $\sim 4-\mathrm{m}$ for telescopes with monolith primary mirrors, and 9 to 11 meters for a segmented primary. While the SLS 8.4-m fairing could arguably accommodate a 15 to 18 meter segmented telescope, there will become a time, as telescope apertures continue to grow, when assembly in space is necessary. For more than a century ground based telescopes have been assembled and serviced on site and continuously upgraded with new instruments to enhance their performance. For example, the 5-m Hale telescope is still highly productive nearly 70-years after First Light, thanks

${ }^{x i}$ While it was in operation, the Space Shuttle frequently operated in this role.

xii But note that a catastrophic failure of the SLS launch system would set back this class of astrophysics systems, probably for decades.

xiii When all seven of the Giant Magellan Telescope's 8.4-m primary mirror segments are in place, the GMT will have the collecting area of a $22-\mathrm{m}$ filled aperture and the resolution of a $24.5-\mathrm{m}$ telescope, almost a thousand times greater than Galileo 
to adaptive optics that have increased it's resolution by a factor 10 to 20 , and CCD detectors that are 20 to 30 times more sensitive than the photographic plates that they replaced ${ }^{\text {xiv }}$.

The feasibility of on-orbit assembly and servicing of large space structures has already been demonstrated by the International Space Station; Efforts to develop the capability to refuel, repair, upgrade and repurpose satellites is LEO and GEO and on-going; and NASA is moving forward on plans for a Deep Space Gateway that could serve as an assembly site for large space vehicles, as well as a site for testing life support systems for Mars Exploration vehicles, a laboratory for testing humans physiological response to long duration flights outside the Earth's magnetosphere in heliocentric space, and a transfer point for travel to the lunar surface.

The development of the 5-m (200-inch) Hale telescope and its predecessors is, in many ways parallels our approach to the development of an Evolvable Space Telescope. The 200-inch is the forth of the large astronomical telescopes developed by George Ellery Hale in the late $19^{\text {th }}$ and early $20^{\text {th }}$ centuries. In 27 years, between 1890 and 1917, Haled developed three telescopes that were the largest in the world at the time of their "first light": the 40-inch refractor at Yerkes Observatory in 1897; the 60-inch Hale telescope at Mt. Wilson in 1908; and Mt. Wilson's 100-in Hooker telescope in 1917. And by 1932 Hale had gotten funding for the 200-inch Mt. Palomar telescope, which finally saw first light in 1948 after work was halted for many years during World War II.

Since the technology required to align and phase the mirrors of a segmented primary was not available at the time, it was necessary for Hale to develop an entirely new telescope at each stage in his quest to create larger and more powerful telescopes. He did, however, use the latest technologies available as his telescopes evolved from the largest possible refractor to ever-larger reflecting telescopes with improved optical materials, and opto-mechanical designs.

As we expect for EST, the scientific discoveries made with Hale's early telescopes drove the design for those that followed. Observations of Cepheid variables led to the discover that nebulae like Andromeda are millions of light years outside our galaxy; and observations of these external galaxies led to Hubble's discovery of the "red shift" that proved our universe is rapidly expanding ${ }^{\mathrm{xV}}$.

Thus a telescope twice the size of the 100-inch Hooker telescope was developed to look deeper into this previously unknown universe. This 200-inch telescope has many features that we have adopted for EST. This includes:

- A compartment at the focus of a relatively slow primary mirror (f/3.3 for the 200 -in) where the sensitivity of instruments is only degraded by a single mirror's reflectivity; and

- Additional locations for instruments provided by mirrors that could be rotated into position in front of the rime focus compartment to provide Cassegrain focus behind the primary mirror (as well as two different Coude foci in the case of the 200-inch telescope)

While previous space telescopes have not had these features, we have adopted them for EST. This includes relatively slow primaries (to minimize polarization effects); a prime focus instrument module (to minimize the number of reflections for coronagraphy and UV spectroscopy); the ability to create a focus behind the primary by rotating a Cassegrain (or Gregorian) secondary mirror onto the optical axis of EST's segmented primary mirror; and, of course, Hale's evolutionary approach to the development of the largest possible telescope(s).

\section{CONCLUSIONS}

- On-orbit assembly and servicing of large space telescopes will be feasible in the 2030s with the necessary infrastructure in place, and these capabilities will be in use for commercial purposes as well as NASA's exploration program.

- Assembly of future large space telescopes in space will "break the 15-m barrier" imposed by the largest payload fairings currently envisioned for Very Large launch vehicles

\footnotetext{
${ }^{\text {xiv }}$ For additional information see $<$ http://www.astro.caltech.edu/palomar/about/telescopes/hale.html\#instruments $>$

${ }^{x v}$ Estimates of the rate of the universe's expansion (Hubble's Constant $-\mathrm{H}_{0}$ ) have varied greatly since Hubble estimated it at $500 \mathrm{~km} / \mathrm{sec}$ in 1929. Observations with the 200-inch telescope, HST, WMAP, Planck and Thee Sloan Digital Sky Survey (SDSS) indicate $\mathrm{H}_{0}$ is $\sim 70 \mathrm{~km} / \mathrm{sec}$ (and that the expansion rate is increasing).
} 
- In an era of flat NASA budgets, building, launching and operating large telescopes in several stages, with increased capability at each stage will:

- Avoid peaks and valleys in the funding requirements that impact other astrophysics programs

- Permit design modifications for each stage to incorporate lessons learned during mission operations, incorporate new technologies, upgrade instruments to

- Enable the insertion of the latest technologies in the observatories telescope and instruments

- Develop an highly experienced work force with efficient manufacturing processes and many years of operations experience before the ultimate 12-m and 20-m LUVOIR telescopes achieve first light

\section{ACKNOWLEDGEMENTS}

Our Evolvable Space Telescope (EST) study was initiated by Northrop Grumman Aerospace Systems in Redondo Beach, California and funded with Internal Research and Development (IRAD) funds from March 2014 through January 20016. Since that date the authors have continued to refine the EST concept using funding from their personal owned organizations. We thank John Mather, Marc Postman, Harley Thronson, Gary Mathews, Mark Clampin, Lee Feinberg, Jeremy Kasdin, Tony Hull, and my others for useful discussions during this study.

\section{REFERENCES}

[1] Polidan, R. S., Breckinridge, J. B., Lillie, C. F., MacEwen, H. A., Flannery, M. R., and Dailey, D. R., "Innovative telescope architectures for future large space observatories", JATIS 2(4), 041211, (2016)

[2] Lee, N., Backes, P., Burdick, J., Pellegrino, S., Fuller, C., Hogstrom, K., Kennedy, B., Kim, J., Mukherjee, R., Seubert, C., and $\mathrm{Wu}, \mathrm{Y}-\mathrm{H} .$, "Architecture for in space robotic assembly of a modular space telescope", JATIS 2(4), 041207, (2016)

[3] Boyd, I. D., Buenconsejo, R. S., Piskorz, D., Lal, B., Crane, K. W., and De La Rosa Blanco, E., “On-Orbit Manufacturing and Assembly of Spacecraft", IDA Science \& Technology Institute, IDA Paper P-8335, (January 2017)

[4] J. Dalcanton et al., "From Cosmic Birth to Living Earths: The Future of UVOIR Astronomy", Association of Universities for Research in Astronomy (AURA), Washington, DC (2015).

[5] MacEwen, H. A., and Lillie, C. F., "Infrastructure for large space telescopes", JATIS (4), 041208 (2016)

[6] Polidan, R. S., Breckinridge, J. B., Lillie, C. F., MacEwen, H. A., Flannery, M. R., and Dailey, D. R., “An Evolvable Space Telescope for Future Astronomical Missions", Proc. SPIE 9143, (2014)

[7] Lillie, C. F., “On-Orbit servicing for Future Space Observatories”, Proc. SPIE 6265, (2006)

[8] Breckinridge, J. B., Kuper, T., and Shack, R. V., "Space Telescope low scattered light camera: a model”. SPIE conference on Instrumentation in Astronomy IV, Tucson AZ. Proc.of the SPIE 331, (1982)

[9] Breckinridge, J. B., Kuper, T., and Shack, R. V., "Space Telescope low-scattered light camera: a model", Optical Engineering 23, 816-820 (1984)

[10] Pueyo, L., Shaklan, S. B., Give'On, A., Troy, M., Kasdin, N. J., et al, “Correction of quasi-static errors for ELT with two sequential DM's", published in conference proceedings: "First conference on Adaptive Optics for Extremely Large Telescopes" DOI: 10.1051/ao4elt/201005009. (2010)

[11] Pueyo, L., Kay J, Kasdin, N. J., Groff T., McElwain M., Give’On A., Belikov R., 'Optimal dark hole generation via two deformable mirrors with stroke minimization”, Applied Optics 48, 6, 296-312, (2009)

[12] Cady, Eric, Prada, C., An, X., Balasubramanian, K., Diaz, Rosemary, Kasdin, N. J., et al, "Demonstration of high contrast with an obscured aperture with the WFIRST-AFTA shaped pupil coronagraph", Journal of Astronomical Telescopes, Instruments, and Systems 2, 011004 (2016)

[13] Mawet, Dimitri, Pueyo, L., Moodya, D., Krist, J., and Serabyn, E., “The Vector Vortex Coronagraph: sensitivity to central obscuration, low-order aberrations, chromaticism, and polarization", (2010)

[14] Murakami, N., Yokochi, K., Nishikawa, J., Tamura, M., "Polarization interferometric nulling coronagraph for highcontrast imaging", Applied Optics 49, D106-114 (2010)

[15] Aime, C., "Radon approach to shaped and apodized apertures for imaging exoplanets", A\&A 434, 785-794, (2005

[16] Guyon O., Pluzhnik, E. A., Galicher, R., et al "Exoplanet imaging with a phase-induced amplitude apodization coronagraph. I. Principle”, ApJ 622, 744-758, (2005)

[17] Breckinridge, J. B., Lam, T. and Chipman, R., "Polarization aberrations in Astronomical Telescopes: The Point Spread Function", Pub. Astr. Soc. Pacific 127, 445-468, (2015) 
[18] Breckinridge, J. B. and Chipman, R. A., "Telescope polarization and image quality: Lyot coronagraph", Proc. SPIE 9904, (2016)

[19] Sabatke E., Burge J., Sabatke, D., "Analytic diffraction analysis of a 32-m telescope with hexagonal segments for high-contrast imaging", Applied Optics 44(8), 1360-1365, (2005)

[20] Bland-Hawthorn, J., and Kern, P., "Molding the flow of light: Photonics in astronomy", Physics Today, 65, 31(2012)

[21] Bland-Hawthorn, J., Lawrence, J., Robertson, G., Campbell, S., Pope, B., et al, "PIMMS: photonic integrated multimode micro spectrograph", Proc. SPIE, 7735, 22, (2010)

[22] Cvetojevic, N., Jovanovic, N., Lawrence, J., Withford, M., Bland-Hawthorn, J., "Developing arrayed waveguide grating spectrographs for multi-object astronomical spectroscopy”, Optics Express, 20, 2062, (2012)

[23] Cvetojevic, N., Jovanovic, N., Betters, C., Lawrence, J. S., Ellis, S. C., Robertson, G., Bland-Hawthorn, J., "First starlight spectrum captured using an integrated photonic micro spectrograph", A\&A, 544, L1, (2012)

[24] Gatkine, P., Veilleux, S., Hu, Y., Zhu, T., Meng, Y., Bland-Hawthorn, J., and Dagenais, M., "Development of highresolution arrayed waveguide grating spectrometers for astronomical applications: first results", Proc. SPIE 9912, $71,(2016)$

[25] Moore, C. S., Hennessy, J., Jewell, A., Nikzad, S., and France, K., "Atomic Layer Deposited (ALD) coatings for future astronomical telescopes: recent developments", Proc. SPIE 9912, (2016)

[26] Stahl, H. P., "Survey of cost models for space telescopes", Optical Engineering 49, Paper 053005, 1-8, (2010)

[27] Breckinridge, J. B., and Lillie C. F., "Prime focus architectures for large space telescopes: reduce surfaces to save cost", Proc. SPIE 9904, (2016,

[28] 2010 Congressional Budget for NASA

[29] NASA, "Hubble servicing missions overview," http://www.nasa.gov/mission pages/hubble/servicing/index.html (17 August 2017)

[30] Kauderer, A., International Space Station Assembly, NASA, (25 March 2011) http://www.nasa.gov/mission_pages/station/structure/iss_assembly.html (17 August 2017)

[31] Lillie, C., and MacEwen, H.; "In-Space Assembly and Servicing Infrastructures for the Evolvable Space Telescope (EST)", Proc. of SPIE 9904, (2016\}

[32] MacEwen, H., Lillie, C., "Infrastructure for large space telescopes," Journal of Astronomical Telescopes, Instruments, and Systems 2(4), 041208, (2016)

[33] Boyd, I., et al, "On-Orbit Manufacturing and Assembly of Spacecraft", IDA Paper P-8335, Institute for Defense Analysis, Alexandria, VA (2017)

[34] Reed, B., "The Restore-L Servicing Mission”, NASA, (29 Mar 2016) http://www.nasa.gov/sites/default/files/atoms/files/reed_restorel_tagged.pdf (17 August 2017)

[35] Reed, B., "SEL2 Servicing: Increased Science Return via On-Orbit Propellant Replenishment”, Proc. of SPIE 9904, (2016)

[36] DARPA, "Program Aims to Facilitate Robotic Servicing of Geosynchronous Satellites", http://www.darpa.mil/news-events/2016-03-25 (2015) (17 August 2017)

[37] Harrison, T., et al, "Implications of Ultra-Low-Cost Access to Space", Center for Strategic and International Studies, Washington, DC, (2017)

[38] NASA, "SLS Program Mission Planners Guide", (August 2014), https://smd-prod.s3.amazonaws.com/sciencered/s3fs public/atoms/files/SLS_Program_Mission_Planners_Guide_Ver1-Aug2014.pdf(17 August 2017)

[39] United Launch Alliance, “Delta IV Launch Services User's Guide”, (June 2013) http://www.ulalaunch.com/uploads/docs/launch_vehicles/delta iv users guide june 2013.pdf (17 August 2017)

40] Arianespace, “Ariane 5 Users Manual”, (October 2016), http://www.arianespace.com/wpcontent/uploads/2011/07/Ariane5_Users-Manual_October2016.pdf (17 August 2017)

[41] SpaceX, "Falcon Heavy: The world's most powerful rocket", (2017) http://www.spacex.com/falcon-heavy (17 August 2017)

[42] Hambleton, K., "Deep Space Gateway to open opportunities for distant destinations”, NASA, (March 28, 2017) https://www.nasa.gov/feature/deep-space-gateway-to-open-opportunities-for-distant-destinations (17 August 2017)

[43] Griffin, B., "Benefits of a single-person spacecraft for weightless operations," in Proc. of the AIAA Int. Conference on Environmental Systems, San Diego, California (2012) 